\title{
CD68+ cell numbers and dendritic cell numbers and phenotype fail to predict the presence of a MYC rearrangement in aggressive B-cell lymphomas
}

\author{
Sandrine Gaslain • Marie Stolbrink • Margaret Jones • \\ Elizabeth J. Soilleux
}

Received: 5 July 2011 / Accepted: 21 November 2011 /Published online: 4 January 2012

(C) Springer-Verlag 2011

\begin{abstract}
MYC rearrangements are frequently associated with aggressive B-cell lymphomas, such as Burkitt's lymphoma. We sought surrogate markers of MYC rearrangements that might obviate the need for a complex and expensive technique such as fluorescent in situ hybridization (FISH). We show that numbers of CD68+ macrophages/dendritic cells (DCs), DC-SIGN+ myeloid DCs and CD123+ plasmacytoid DCs fail to correlate with MYC rearrangement, although a starry sky appearance shows positive correlation $(p=0.003)$ when statistical analysis is performed on all three diagnostic groups together. We conclude that FISH is required to investigate the presence of a MYC rearrangement in cases of aggressive B-cell lymphoma, in order to guide the chemotherapeutic regime offered to the patient.
\end{abstract}

Keywords Burkitt's lymphoma · Diffuse large B-cell lymphoma - MYC - Translocation - Rearrangement - Histiocyte . Macrophage · Dendritic cell · CD68 · DC-SIGN · CD123

S. Gaslain · E. J. Soilleux $(\bowtie)$

Department of Cellular Pathology, John Radcliffe Hospital,

Oxford OX3 9DU, UK

e-mail: elizabeth.soilleux@ndcls.ox.ac.uk

M. Stolbrink

Oxford University Medical School,

Oxford, UK

M. Jones $\cdot$ E. J. Soilleux

Nuffield Department of Clinical Laboratory Sciences,

University of Oxford,

Oxford OX3 9DU, UK

\section{Introduction}

The major types of aggressive B-cell lymphoma (BCL) are Burkitt's lymphoma (BL) and diffuse large B-cell lymphoma (DLBCL). BL has specific starry sky morphology, consequent upon macrophages taking up apoptotic debris. It is almost always associated with MYC rearrangement and often with Epstein-Barr virus (EBV), although a MYC rearrangement is not specific to $\mathrm{BL}$. It has a high proliferation index (PI) and characteristic CD10+ bcl-2- TdT- immunophenotype [1]. DLBCL encompasses most other aggressive BCLs with a PI $>40 \%$. In general, DLBCL is less aggressive than BL. An intermediate category "B-cell lymphoma, unclassifiable, with features intermediate between DLBCL and Burkitt lymphoma" (IBLDBCL) between the two exists. The majority of cases in this category have morphological features that are intermediate between DLBCL and BL with some cells that are smaller than typical DLBCL, resembling BL, and some cells that are larger than typical BL, resembling DLBCL, as well as a high proliferation fraction, starry sky pattern, and an immunophenotype consistent with BL. In addition to BL, a significant proportion of IBLDBCL and a smaller proportion of DLBCL carry a MYC translocation. These tumours usually have a high PI, often $>90 \%$ [1]. BL lacking demonstrable MYC rearrangements and DLBCL with MYC rearrangements should not be included in the IBLDBCL diagnostic category, meaning that almost all cases will be assigned to this diagnostic category on the basis of a combination of morphological and immunohistochemical features [1]. Notwithstanding, a recent publication suggests a three-phase approach may be helpful in the separation of these categories. In this approach, phase 1 involves assessment of 
morphology with CD10 and BCL2 immunostains; phase 2 employs CD38, CD44, and Ki-67 immunostains and phase 3 entails fluorescent in situ hybridization (FISH) on paraffin sections for MYC, BCL2, BCL6 and immunoglobulin family genes [2]. However, this approach has yet to be adopted as the "gold standard" by the WHO [1]. Increasingly, MYC+ BCLs are treated with a specialised chemotherapeutic protocol, CODOX-M (cyclophosphamide, vincristine (Oncovin), doxorubicin and methotrexate), rather than the CHOP-R protocol (cyclophosphamide, doxorubicin (hydroxydaunorubicin), oncovin, prednisolone and rituximab) normally used for DLBCL [3]. MYC rearrangements can be demonstrated by FISH, a relatively expensive, specialised and time-consuming technique [3]. We investigated whether the numbers of macrophages or dendritic cells (DCs) of plasmacytoid (pDC) or myeloid (mDC) type could be used as a surrogate marker to predict the presence of MYC rearrangements.

Macrophages and the majority of DCs are CD68+ [4]. DCs are specialised antigen-presenting cells capable of initiating immune responses. The DC component in a tumour may be important in determining aggressiveness and prognosis [5]. As aggressive BCLs with a MYC rearrangement carry a poorer prognosis than those without [1], we wondered whether different complements of DCs might be found in BL and DLBCL. DCs may be divided into myeloid and plasmacytoid types. Outside secondary lymphoid tissue, such as lymph nodes, tonsil and spleen, DCs are generally immature, requiring particular signals to become competent to activate naïve $\mathrm{T}$ cells and induce immune responses. Relatively specific markers are available for immature $\mathrm{mDC}$ and $\mathrm{pDC}$ and here we use DC-SIGN (mDC) and CD123 (pDC) [4]. We fail to demonstrate any correlation between the presence of a MYC rearrangement and the number of cells positive for CD68, DCSIGN or CD123, but demonstrate correlation between a starry sky appearance and MYC rearrangement.

\section{Materials and methods}

Tissue was obtained with full ethical approval from the National Research and Ethics Service (ref: C02.162) from the Department of Cellular Pathology, John Radcliffe Hospital, Oxford, from cases of CD10+ bcl-2- PI $>90 \%$ Burkitt's lymphoma $(n=8)$, CD10+ bcl-2+ PI $>90 \%$ diffuse large B-cell lymphoma $(n=10)$ and $\mathrm{CD} 10+$ bcl-2- PI $>90 \%$, IBLDBCL $(n=11$, Table 1$)$. The IBLDBCL cases demonstrated an immunophenotype, but not a morphology, compatible with Burkitt lymphoma. No cases with Burkitt morphology and $\mathrm{CD} 10+$ bcl-2+ PI $>90 \%$ phenotype (which would also count as IBLDBCL) were identified in our archives. To avoid selection bias, all cases in our diagnostic archive from the last 10 years that fitted the above criteria and where adequate tissue remained were used in this study.
All cases had been reviewed by two or three specialist consultant haematopathologists at the time of diagnosis, and only cases with full agreement between all reviewing pathologists were used in the study.

Haematoxylin and eosin staining was performed followed by immunostaining for CD68 (clone PGM1, a generous gift from Professor Kevin Gatter, Nuffield Department of Clinical Laboratory Sciences, Oxford University, UK) [6], DC-SIGN (clone DC28; R\&D Systems, Abingdon, UK) [4], and CD123 (clone 107D2.08; Schering Plough, Welwyn Garden City, UK) [7] using the automated BondMax ${ }^{\mathrm{TM}}$ staining system (Leica Microsystems, Wetzlar, Germany), as per the manufacturers' instructions. For all three antibodies, antigen retrieval was performed using the high pH Epitope Retrieval 2 solution (Leica Microsystems, Wetzlar, Germany) at $100^{\circ} \mathrm{C}$ for $20 \mathrm{~min}$. This was followed by a 5-min peroxidase block as per the manufacturers' kit and instructions (Leica Microsystems, Wetzlar, Germany). Numbers of positively immunostained cells were counted in five representative high power $(\times 400)$ microscope fields in each tumour, and the intensity of staining was also recorded on a scale of 1-3 where 3 was most intense. The DC28 antibody cross-reacts with DC-SIGN and DC-SIGNR [4], both of which are expressed on lymph node sinus endothelial cells [8]. These were identified as a continuous line of cells and excluded from cell counts. CD123+ vascular endothelial cells [9] were similarly excluded from cell counts.

In situ hybridisation for EBV with the EBER probe (Leica Microsystems, Newcastle, UK) was performed using the BondMax ${ }^{\mathrm{TM}}$ staining system (Leica Microsystems, Wetzlar, Germany), as per the manufacturers' instructions. Fluorescent in situ hybridisation (FISH) was performed, using Vysis LSI MYC Dual Color Break Apart probe 30191096 (Abott Molecular, Maidenhead, UK), as per the manufacturers' instructions [10]. Slides were examined using a Zeiss Axioskop microscope with an excitation filter wheel (Carl Zeiss Ltd., Welwyn Garden City, UK) and a Hamamatsu Orca C4742-95-12NRB camera (Hamamatsu Photonics UK Ltd., Welwyn Garden City, U.K.). For each case, 100 nuclei were scored for the presence of a rearrangement and deemed positive if a rearrangement was identified in $>20 \%$ cells [11]. Statistical analysis was performed using SPSS Statistics 17.0. (SPSS Inc., Chicago, IL, USA) and the nonparametric Kruskal-Wallis test or Fisher's exact test was used in the generation of all $p$ values using a 95\% confidence level.

\section{Results}

A starry sky appearance was present in 6 out of 7 BL cases (poorly preserved morphology made this difficult to judge 


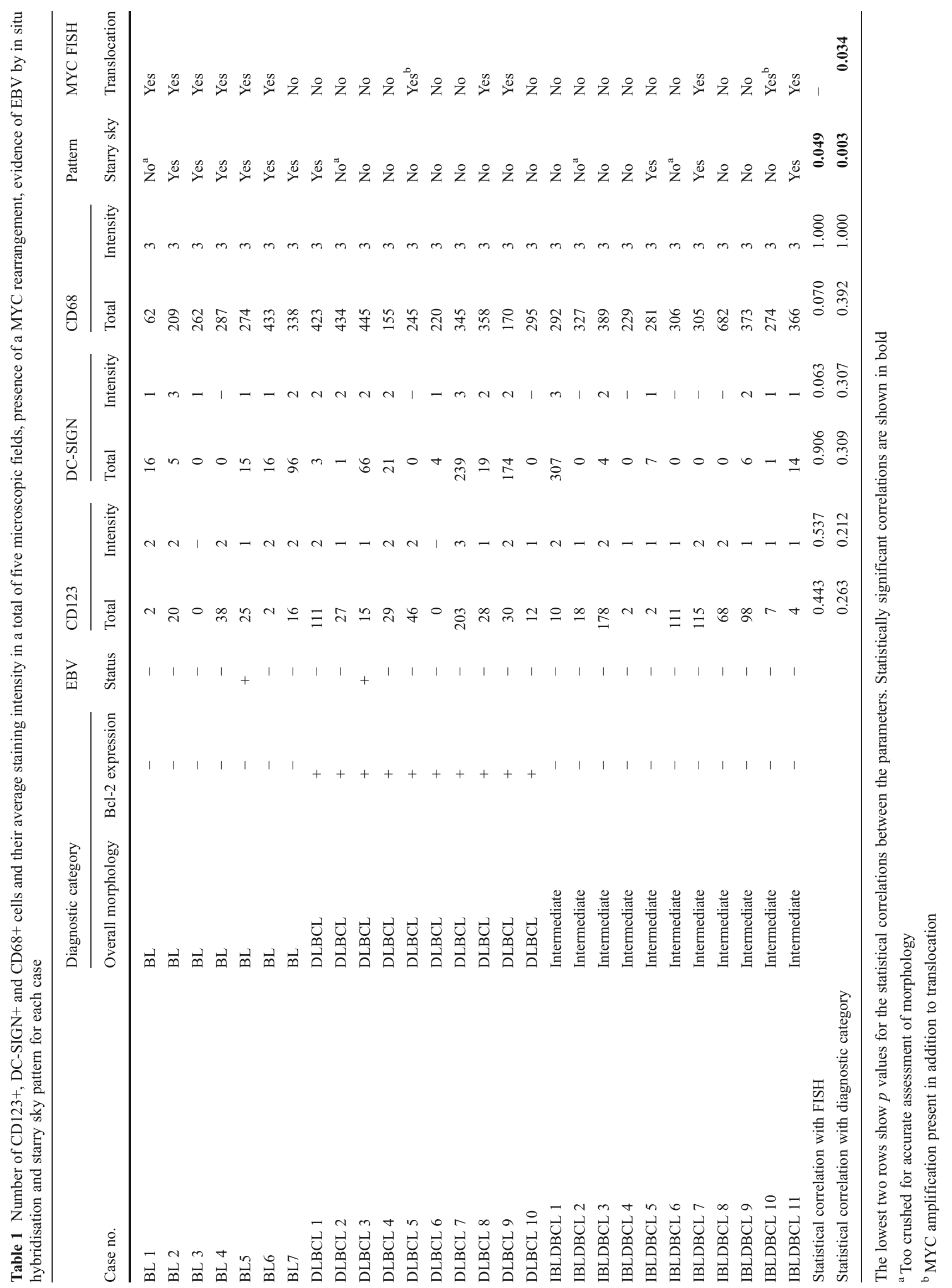


in 1 out of 7), 0 out of 10 DLBCL cases and in 3 out of 11 IBLDBCL cases, although 2 further IBLDBCL cases had poorly preserved morphology (Table 1). Figure 1a
(CD68 immunostaining) demonstrates the presence of abundant large macrophages in BL. FISH using MYC break-apart probes demonstrated a translocation in 6 out

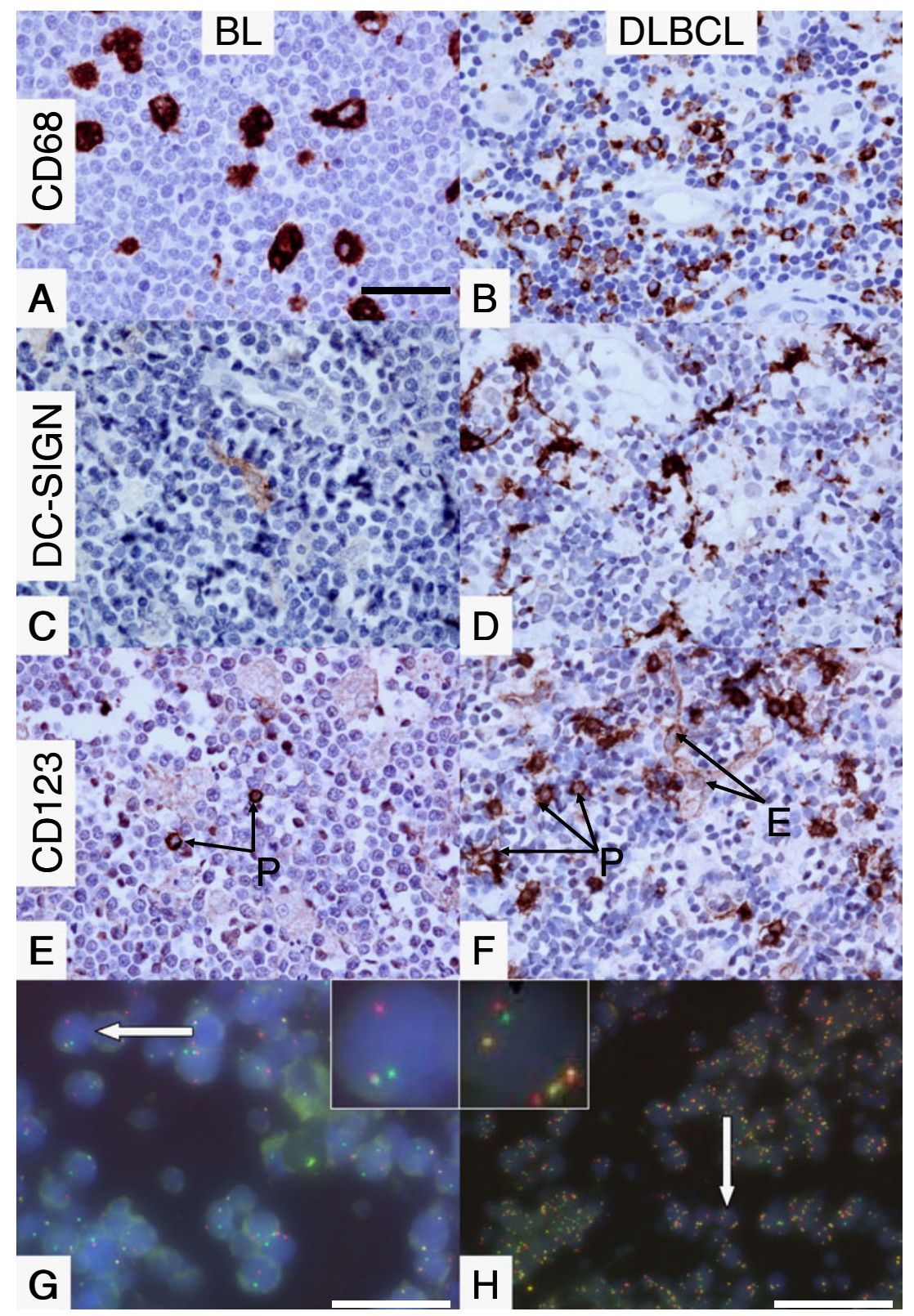

Fig. 1 Macrophage and dendritic cell markers $(\mathbf{a}-\mathbf{f})$ immunostained using the peroxidase technique (brown). CD68 immunostaining was strong on any positive cells (Fig. 1a, b). In BL, the vast majority of CD68+ cells were large round cells with macrophage morphology, containing apoptotic debris. CD68+ cells in DLBCL (b) and most IBLDBCL were smaller with either plasmacytoid or dendritic morphology, in keeping with a mixture of macrophages, $\mathrm{pDC}$ and $\mathrm{mDC}$. In all three categories, the immature mDC marker, DC-SIGN, immunostained very variable numbers of cells with dendritic morphology relatively weakly, as demonstrated by the central light brown cell with dendritic morphology in c. DLBCL generally showed stronger immunostaining and, in this case, DCs are more abundant (d). The pDC marker, CD123, immunostained variable numbers of small- to medium-sized round cells with weak or intermediate intensity, highlighting smaller numbers of cells in the BL (e) than the IBLDBCL or DLBCL groups (f). pDC are marked $\mathrm{P}$ and vascular CD123+ endothelial cells (not counted) are marked E. FISH using MYC break-apart probes shows two fused red-green (yellow) signals in normal cells, but allows separate red and green signals to be visualized if there is a translocation. There was a translocation in $6 / 7$ BL cases, as in the case in $\mathbf{g}$ (good example shown with an arrow and magnified in top right hand corner). There was a MYC translocation in 3 out of 10 DLBCL cases, one of which (h) additionally demonstrated MYC amplification (good example shown with an arrow and magnified in top left hand corner). Scale bar in a (50 microns) refers to panels a-f. Scale bars in $\mathbf{g}$ and $\mathbf{h}$ are 20 and 40 microns, respectively 
of $7 \mathrm{BL}$ cases, but showed no abnormality in 1 out of 7 (Table 1, Fig. 1g). There was a MYC translocation in 3 out of 10 DLBCL cases, 1 additionally demonstrating MYC amplification (Table 1, Fig. 1h). Three out of 11 IBLDBCL cases demonstrated a MYC translocation, 1 with an additional MYC amplification (Table 1). EBV RNA expression was detected in only 1 out of 7 BL cases and 1 out of 10 DLBCL cases (Table 1).

CD68 immunostaining was strong on all positive cells (Fig. 1a, b). In BL, the vast majority of CD68+ cells were large round cells with macrophage morphology, containing apoptotic debris. CD68+ cells in DLBCL and most IBLDBCL were smaller with either plasmacytoid or dendritic morphology, in keeping with a mixture of macrophages, $\mathrm{pDC}$ and $\mathrm{mDC}$. In all three categories, the immature mDC marker, DC-SIGN, immunostained very variable numbers of cells with dendritic morphology relatively weakly, although DLBCL generally showed stronger immunostaining (Fig. 1c, d). The pDC marker, CD123, immunostained variable numbers of cells with weak or intermediate intensity in the IBLDBCL and DLBCL groups, but generally immunostained only small numbers of cells in BL cases.

There was no statistically significant correlation between the numbers or staining intensity of CD68+, DC-SIGN+ or $\mathrm{CD} 123+$ cells and either the presence of a MYC rearrangement or the diagnostic category, with $p$ values $>0.05$ (Table 1). In contrast, starry sky morphology correlated significantly with MYC rearrangement $(p=0.003)$. The diagnostic category of lymphoma also correlated with MYC rearrangement, with $\mathrm{BL}$ predicting a rearrangement $(p=0.034)$. These two expected statistical correlations validate the study and suggest that adequate numbers of cases were used to detect any strong statistical correlations between CD123+, CD68+ and DC$\mathrm{SIGN}+$ cell numbers/staining intensity and MYC rearrangement or diagnostic category. However, it is possible that weaker correlations could only be detected by using a larger number of cases.

In light of the correlation between a starry sky morphological pattern and a MYC rearrangement when all the diagnostic categories were analysed together, we investigated whether a starry sky pattern alone could be used to predict the presence of a MYC rearrangement within the IBLDBCL diagnostic category. A starry sky morphological pattern alone did not demonstrate statistically significant correlation with the presence of a MYC rearrangement $(p=0.09)$.

\section{Discussion}

We investigated whether the numbers of macrophages, pDCs or mDCs could be used as a surrogate marker to predict the presence of MYC rearrangements and showed that they could not. However, we demonstrated statistical correlation between both starry sky morphology and the diagnostic category of lymphoma and the presence of a MYC rearrangement. Interestingly, narrowing the analysis to within the IBLDBCL diagnostic category, a starry sky morphological pattern alone did not demonstrate statistically significant correlation with the presence of a MYC rearrangement $(p=0.09)$.

FISH studies demonstrated a MYC rearrangement in all but 1 out of 7 cases of BL. This is consistent with reports suggesting that in up to $10 \% \mathrm{BL}$ cases, no MYC translocation is demonstrable by FISH, the precise explanation for which remains uncertain $[1,12,13]$. A MYC rearrangement was identified in only in 3 out of $11(27 \%)$ IBLDBCL cases, which, given the small size of the present study, is in keeping with other studies suggesting that 35-50\% IBLDBCL cases carry a MYC rearrangement [1, 12-14].

A simple explanation for the correlation between the starry sky pattern and the presence of a MYC rearrangement is Myc's ability to trigger rapid apoptosis, as well as cell proliferation. Deregulated Myc expression indirectly induces accumulation and activation of the tumour suppressor p53. Increased p53 activity then transactivates genes encoding pro-apoptotic proteins, such as Bax, Bid, Noxa and Puma, and/or initiates release of cytochrome c from mitochondria to activate the apoptotic machinery [15]. Abundant macrophages take up apoptotic debris, giving typical starry sky morphology [1].

The fact that CD68 is expressed by DCs as well as macrophages may have obscured any correlation between the numbers of macrophages and the presence of a MYC rearrangement. It may, therefore, have been helpful to use a more macrophage-specific marker, such as CD14, although this can also be expressed by certain DC subsets [4]. Due to the degree of overlap between macrophage and DC phenotypes, we did not try to identify macrophages as a distinct category from DCs by immunostaining. The factors determining the numbers of DCs of particular phenotypes present in any tissue, including a tumour, are complex and may include the cytokine milieu and adhesion molecule expression by the tumour and its microenvironment [16], which may be entirely unrelated to the PI, rate of apoptosis or presence of a MYC rearrangement.

Although BL is a relatively homogeneous entity, the current DLBCL category is by no means homogeneous and may be divided into two or three major subgroups [17] by immunohistochemistry or gene expression profiling. However, given our selection of CD10+ DLBCLs, it is likely that, although still not an entirely homogeneous group, all the DLBCL and IBLDBCL cases would have fallen into the germinal centre $B$ cell rather than the activated B-cell subgroup. While the cases in our IBLDBCL category were as homogeneous as possible on morphological and 
immunohistochemical grounds, the demonstration of a MYC rearrangement in only 3 out of 11 cases suggests that this category is not particularly homogeneous. Some IBLDBCL cases that carry a MYC rearrangement may also carry a second chromosomal rearrangement ("double hit lymphoma"), usually of BCL2 [18]. Nevertheless, the IBLDBCL category is still useful for aggressive B-cell lymphomas with some features of Burkitt lymphoma, not least because all IBLDBCL cases should probably be investigated for MYC rearrangements. MYC rearranged IBLDBCL cases may then be treated with CODOX-M rather than CHOP-R.

This is a small and preliminary study, and if repeated on a larger patient cohort, it may demonstrate some weaker statistical correlations undetectable in the present study. Such a study would also allow analysis of the predictive ability of combinations of morphological, immunohistochemical and genetic features in the separation of these lymphomas into diagnostic groups. There are various difficulties obtaining larger numbers of cases. Firstly, the diagnosis of BL and IBLDBCL is relatively uncommon. Secondly, many diagnostic specimens in such cases are small core biopsies, particularly if patients are relatively unwell at diagnosis, for example precluding general anaesthetic. This frequently means that inadequate tissue remains after diagnosis for a study such as ours. Nevertheless, this study showed a lack of correlation between a MYC rearrangement and the types and numbers of DCs and macrophages present in the tumours, DCs and macrophages, suggesting that they cannot be used as surrogate markers for MYC rearrangement. Despite being a relatively expensive, technically complex and time-consuming technique, we would continue to advocate the use of FISH to look for the presence of a MYC rearrangement in CD10+ aggressive B-cell lymphomas with a high PI to ensure that patients are categorised appropriately for treatment purposes.

Acknowledgements This project was funded by the BiomedicaI Research Council grants from the National Institute for Health Research. We thank Miss D. Bailey and Mr P. Allen for their assistance with the immunohistochemical staining.

Conflict of interest The authors state that they have no conflict of interest.

\section{References}

1. Swerdlow SH, Campo E, Harris NL et al (2008) WHO classification of tumours of haematopoietic and lymphoid tissues. IARC, Lyon
2. Naresh KN, Ibrahim HA, Lazzi S et al (2011) Diagnosis of Burkitt lymphoma using an algorithmic approach-applicable in both resource-poor and resource-rich countries. Br J Haematol 6:770 776

3. Mead GM, Barrans SL, Qian W et al (2008) A prospective clinicopathologic study of dose-modified CODOX-M/IVAC in patients with sporadic Burkitt lymphoma defined using cytogenetic and immunophenotypic criteria (MRC/NCRI LY10 trial). Blood 112:2248-2260

4. Soilleux EJ, Morris LS, Leslie G et al (2002) Constitutive and induced expression of DC-SIGN on dendritic cell and macrophage subpopulations in situ and in vitro. J Leukoc Biol 71:445-457

5. Soilleux EJ, Rous B, Love K et al (2003) Myxofibrosarcomas contain large numbers of infiltrating immature dendritic cells. Am J Clin Pathol 119:540-545

6. Kunisch E, Fuhrmann R, Roth A et al (2004) Macrophage specificity of three anti-CD68 monoclonal antibodies (KP1, EBM11, and PGM1) widely used for immunohistochemistry and flow cytometry. Ann Rheum Dis 63:774-784

7. Pulford K, Banham AH, Lyne L et al (2006) The BCL11AXL transcription factor: its distribution in normal and malignant tissues and use as a marker for plasmacytoid dendritic cells. Leukemia 20:1439-1441

8. Soilleux EJ, Morris LS, Rushbrook S et al (2002) Expression of human immunodeficiency virus (HIV)-binding lectin DC-SIGNR: consequences for HIV infection and immunity. Hum Pathol 33:652-659

9. Lim LH, Burdick MM, Hudson SA et al (2006) Stimulation of human endothelium with IL-3 induces selective basophil accumulation in vitro. J Immunol 176:5346-5353

10. van Rijk A, Mason D, Jones M et al (2008) Translocation detection in lymphoma diagnosis by split-signal FISH: a standardised approach. J Hematop 1:119-126

11. Ventura RA, Martin-Subero JI, Jones M et al (2006) FISH analysis for the detection of lymphoma-associated chromosomal abnormalities in routine paraffin-embedded tissue. J Mol Diagn 8:141-151

12. Haralambieva E, Boerma EJ, van Imhoff GW et al (2005) Clinical, immunophenotypic, and genetic analysis of adult lymphomas with morphologic features of Burkitt lymphoma. Am J Surg Pathol 29:1086-1094

13. Hummel M, Bentink S, Berger H et al (2006) A biologic definition of Burkitt's lymphoma from transcriptional and genomic profiling. N Engl J Med 354:2419-2430

14. McClure RF, Remstein ED, Macon WR et al (2005) Adult B-cell lymphomas with Burkitt-like morphology are phenotypically and genotypically heterogeneous with aggressive clinical behavior. Am J Surg Pathol 29:1652-1660

15. Allday MJ (2009) How does Epstein-Barr virus (EBV) complement the activation of Myc in the pathogenesis of Burkitt's lymphoma? Semin Cancer Biol 19:366-376

16. Fiore F, Von Bergwelt-Baildon MS, Drebber U et al (2006) Dendritic cells are significantly reduced in non-Hodgkin's lymphoma and express less CCR7 and CD62L. Leuk Lymphoma 47:613-622

17. Choi WW, Weisenburger DD, Greiner TC et al (2009) A new immunostain algorithm classifies diffuse large B-cell lymphoma into molecular subtypes with high accuracy. Clin Cancer Res 15:5494-5502

18. Aukema SM, Siebert R, Schuuring E et al (2011) Double-hit B-cell lymphomas. Blood 117:2319-2331 